\title{
Evidence for separate representations for action and location in implicit motor sequencing
}

\author{
JESSICA K. WITT and DANIEL T. WILLINGHAM \\ University of Virginia, Charlottesville, Virginia
}

\begin{abstract}
We examined sequential learning of actions in an experiment in which four different actions (push, twist, pinch, switch) were placed at four horizontal locations. At transfer, participants responded to a sequence that required performing the same sequence of actions at different locations and to a different sequence of actions at the same sequence of locations. Participants with explicit knowledge demonstrated only learning the sequence of response locations. However, participants with implicit knowledge learned the sequence of actions just as well as the sequence of locations, and performance on individual sequences was just as good as performance when both sequences were presented. These results demonstrated that two types of sequences, one of actions and another of response locations, can be learned simultaneously, suggesting that parallel representations are involved in implicit motor skill acquisition.
\end{abstract}

Skilled actions in everyday life require sequential knowledge in order to prepare responses in advance. Motor skill learning has often been explored in the lab using the serial response time (SRT) paradigm (Nissen \& Bullemer, 1987). In this task, participants respond to stimuli that, unbeknownst to the participants, sometimes appear in a repeating sequence and other times appear randomly. Learning is assessed in two ways. One measure is the time it takes to respond to the sequenced stimuli relative to random stimuli; this measure is considered implicit because it is based on performance and is not associated with awareness of the sequence. The second measure is an interview, or recognition or recall measure. These measures are considered explicit because participants know that their memory is being tapped, and performance is necessarily associated with awareness of the material remembered. Interestingly, response times (RTs) often decrease even though the participant is unaware of the sequence (Willingham, Nissen, \& Bullemer, 1989). Some researchers claim that the two types of learning represent the workings of different memory systems (e.g., Squire \& ZolaMorgan, 1991), which raises the possibility that the two systems rely on different types of representations.

Most investigations on the nature of the representation for motor skill acquisition have used buttonpressing as the response, and it is well established that people are capable of learning sequences of response locations implicitly (e.g., Nattkemper \& Prinz, 1997; Willingham, Wells, Farrell, \& Stemwedel, 2000; but see Remillard, 2003). This

This research was supported by NIH Grants R01 NS040106-05 and R01 MH065598-01A1 to the second author. The authors thank Jason Augustyn for his comments on an earlier draft. Correspondence concerning this article should be addressed to D. T. Willingham, University of Virginia, P.O. Box 400400, Charlottesville, VA 22904 (e-mail: willingham @virginia.edu). task provides a useful way to study skills, such as piano playing and typing, for which the actions (keypresses) vary little and responses are differentiated by spatial location. However, other types of motor skills require learning a sequence of different actions. For example, dancers and martial artists learn sequences of different kinds of movements; however, this learning is usually explicit. To our knowledge, no research has investigated whether action sequences can be learned implicitly. By action sequence, we mean a sequence in which the responses can be differentiated by the action required to make the response. In the typical implicit SRT tasks, participants respond with the same action throughout the sequence (keypresses), so the sequence cannot be learned as a sequence of distinct actions and, rather, is typically learned as a sequence of response locations.

To address whether action sequences can be learned implicitly, we used a modified SRT task. Participants responded to spatial stimuli on a response board with four manipulanda, each requiring a unique action. At transfer, the participants responded on a different board in which the manipulanda were placed at different spatial locations. They responded to a sequence that required performing the same sequence of actions at different locations and a sequence that required performing the same sequence of response locations but with a different sequence of actions.

\section{METHOD}

\section{Participants}

Thirty-six students ( 21 females, 15 males) at the University of Virginia participated in exchange for course credit or payment. All participants gave informed consent.

\footnotetext{
Stimuli and Apparatus

The participants responded to stimuli on two different response boards. Both response boards had a row of four different types of responses: a knob that turned, a button that pressed down, two metal pieces that could be pinched together, and a toggle switch. From left
} 
to right, the response actions on the training response board were press, turn, pinch, and switch. The responses on the test response board were pinch, switch, turn, and press. The distance between the center of each response action was $9.1 \mathrm{~cm}$.

The stimuli consisted of a row of four squares, each $2.3 \mathrm{~cm}$ wide and spaced $3.1 \mathrm{~cm}$ apart. On each trial, a $1.3-\mathrm{cm}$-diameter circle appeared in one of the four squares to indicate which location to make a response. Responses were spatially compatible, so a circle in the far left square required a response to the far left location.

Each participant saw one of four 12-unit sequences. These sequences were chosen to minimize the repetition of the same sequence of actions in training as in test when presented with the same sequence of spatial locations. As an example of an undesirable sequence, 34124 would result in the sequence of pinch, switch, and press in both training (spatial positions 341 ) and test (spatial positions 124). The following sequences were used: 124312134243 , 432143412321, 213421243134, and 341234321412.

The random trial block followed four constraints: a spatial location could not be repeated, each location appeared at the same frequency as the others, a sequence of two locations could not be immediately repeated (1313 was not allowed), and the locations could not increase sequentially over 4 (1234 was not allowed).

\section{Design}

The experiment consisted of nine blocks of stimuli. For the first six blocks, the participants responded on the training response board. After six blocks, they switched to the test response board for the remaining three blocks. There were two types of blocks. Random blocks consisted of 108 random stimuli, and sequenced blocks consisted of a 12-unit sequence followed by six random stimuli, which was repeated six times for a total of 108 trials. There were two reasons for alternating between the 12-unit sequence and 6 units of random stimuli. First, we can compare performance on the sequence to performance on random stimuli within an individual block rather than across different blocks. Second, we thought there would be less explicit knowledge of the sequence because there is only a single presentation of the sequence before the participants see random stimuli again. The first six blocks were all sequenced blocks after which the participants switched to the test response board. The remaining three blocks consisted of one block of random stimuli and two final test blocks. In one of the test blocks, the stimulus sequence was the same as in training, so the participants responded to the same sequence of locations but the sequence of actions was different (same-locations test). In the other test block, the sequence was reorganized so that the sequence of actions was the same as in training but the sequence of locations was different (same-actions test). Test order was counterbalanced across subjects: 18 participants saw the same-locations test first and 18 saw the same-actions test first.

\section{Procedure}

The participants were instructed on how to use the manipulanda. Repeating sequences were not mentioned, and the participants were told to respond as quickly as possible without making errors. Errors were signaled by a 1,000 -msec tone, and the stimulus remained until a correct response was made. The response to stimulus interval was $400 \mathrm{msec}$, and there was a 45 -sec rest break between blocks.

After the participants had completed all nine blocks, we gave them a survey to test explicit knowledge. First, they indicated whether they had definitely seen a sequence, might have seen a sequence, probably did not see a sequence, or definitely did not see a sequence. Then they were asked to write down as much of the sequence as possible. They were allowed to list the sequence of actions or to use the numbers $1-4$ to represent locations.

\section{RESULTS}

Due to a programming error, the rules governing the random trials (e.g., that a stimulus position could not im- mediately repeat) were sometimes violated. We removed all violations and the trial immediately following a violation. Overall, this constituted less than $1.7 \%$ of the data. We also removed trials on which the participants made an error. Overall, accuracy was higher than $96 \%$ and will not be discussed further.

To summarize RTs, we took the median RT for each set of 12 sequenced stimuli and another for each set of 6 random stimuli. We then calculated the mean of these medians for each block. RTs were calculated in an analogous manner for the random block: We took the median for each set of 12 random stimuli and the median for each set of the following 6 random stimuli, and we calculated the means of these medians for each block. These RTs are plotted in Figure 1.

In order to assess learning, we used RT differences between random and sequenced stimuli within a block as the performance measure of sequence learning. Performance on Block 6 revealed that the participants showed reliable learning by the end of training $[t(35)=8.381$, $p<.001]$.

The critical question for this experiment was whether knowledge of the two sequences when learned together transferred to the tests in which only one of the sequences was present. We compared performance measures on each test (same locations and same actions) to the last block of training (training sequence). A repeated measures analysis of the three tests revealed a significant difference $\left[F(2,70)=5.49, M S_{\mathrm{e}}=1,687, p<.01\right]$. Performance on the same-locations sequence was just as good as on the training sequence $[F(1,35)=0.23, p=.63]$ and better than performance on the same-actions sequence $\left[F(1,35)=6.65, M S_{\mathrm{e}}=3,458, p<.05\right]$. Performance on the same-actions sequence was worse than performance on the training sequence $\left[F(1,35)=8.48, M S_{\mathrm{e}}=3,745, p<\right.$ $.01]$. These results suggest that the participants learned the sequence of response locations better than the sequence of actions (see Figure 2). However, a $t$ test revealed that the participants did show some learning of the sequence of actions $[t(35)=4.10, p<.001]$, suggestive of at least partial transfer. Performance on the same-locations sequence was also significantly greater than $0[t(35)=5.74$, $p<.001]$.

A different pattern of results emerged, however, when we analyzed the participants who gained explicit knowledge separately from those who did not. To determine explicit knowledge, we compared free recall with the sequences presented during the same-locations test and the same-actions test. For each test, explicit knowledge was calculated as the number of correctly recalled units of the sequence. Recall did not have to be entirely consecutive; the participants could recall different parts of the sequence. A recalled section had to be at least 3 units in a row, and the explicit score was the sum of all the recalled sections that were 3 or more units long.

Each participant got two explicit scores: one for the same-locations test $(x=3.81, S D=3.4)$ and one for the same-actions test $(x=0.92, S D=1.5)$. A repeated measures ANOVA revealed that the participants recalled the 


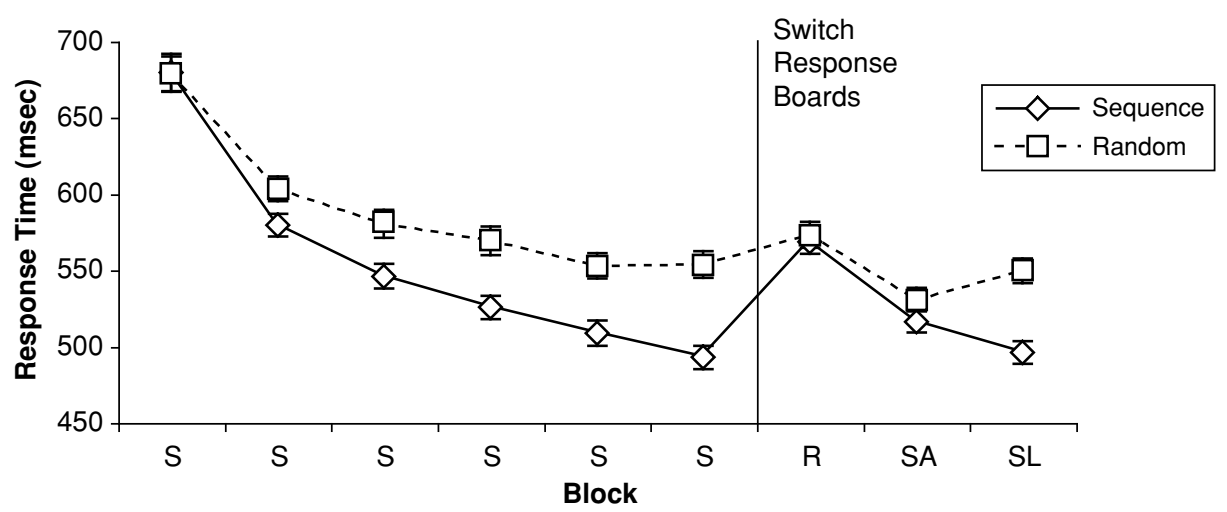

Figure 1. Summarized response times for each block. The vertical line indicates when the participants switched response boards. Error bars depict standard errors of the means. S, block containing alternating 12 unit sequence and 6 units of random stimuli; $R$, block containing only random stimuli; SA, test block in which the sequence preserves the sequence of actions but the sequence of locations is different; SL, test block in which the sequence preserves the sequence of locations but the sequence of actions is different. The order of SA and SL were counterbalanced in the actual experiment. For the random block, both data points are based on response times to random stimuli.

same-locations sequence better than the same-actions sequence $\left[F(1,35)=23.92, M S_{\mathrm{e}}=6.28, p<.001\right]$. Since few participants had any explicit knowledge of the sameactions sequence, we divided the participants into two groups on the basis of how much explicit knowledge they had of the same-locations sequence. Anyone who recalled $50 \%$ or more of the same-locations sequence was considered to have explicit knowledge $(n=14)$, and everyone else was considered to have only implicit knowledge $(n=$ 22). ${ }^{1}$

As shown in Figure 3, the participants with implicit knowledge showed a different pattern of results than did the participants with explicit knowledge of the locations sequence. The participants with implicit knowledge learned both the sequence of response locations and the sequence of response actions. A repeated measures ANOVA with test as a within-participants factor revealed that there were no differences in performance across the three tests for the implicit group $[F(2,42)=0.24, p=$ .79]. Individual $t$ tests revealed that performance on all three tests was greater than 0 [training sequence, $t(21)=$ $6.90, p<.001$; same locations, $t(21)=4.48, p<.001$; same actions, $t(21)=3.99, p<.001]$. Thus, when only the sequence of actions or the sequence of locations was present, the participants' performance was equivalent and was no better or worse than when both sequences were present.

In contrast, the participants with explicit knowledge of the locations sequence did not demonstrate learning of the sequence of actions (see Figure 3). A repeated measures ANOVA with test as a within-participants factor revealed a significant difference between the three tests $[F(2,26)=$ 13.63, $\left.M S_{\mathrm{e}}=1,231, p<.001\right]$. Planned contrasts showed that performance on the same-actions sequence was worse

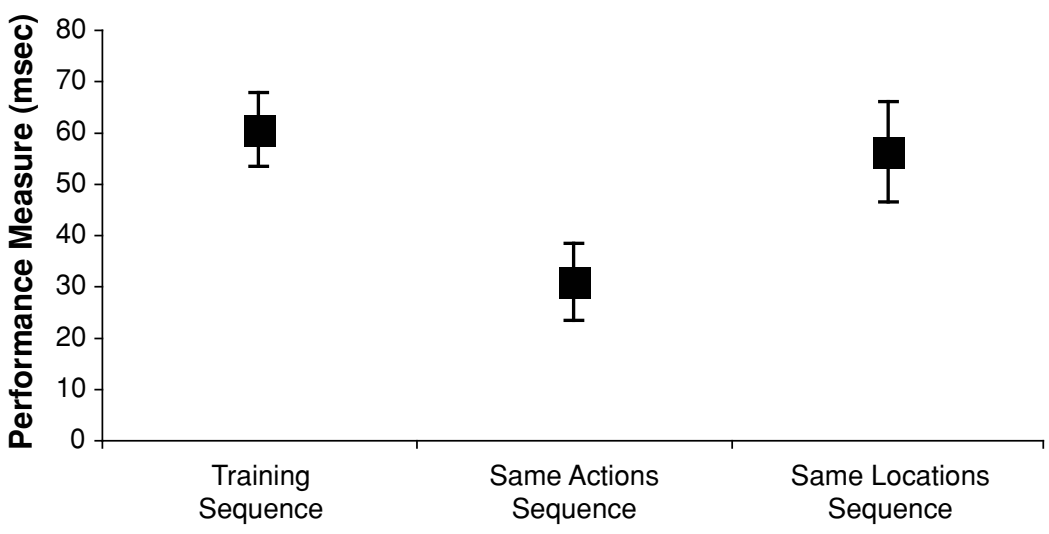

Figure 2. Performance on the three test conditions. The performance measure indicates the response time on the sequenced stimuli subtracted from response time on the random stimuli within the same block. Error bars depict standard errors of the means. 


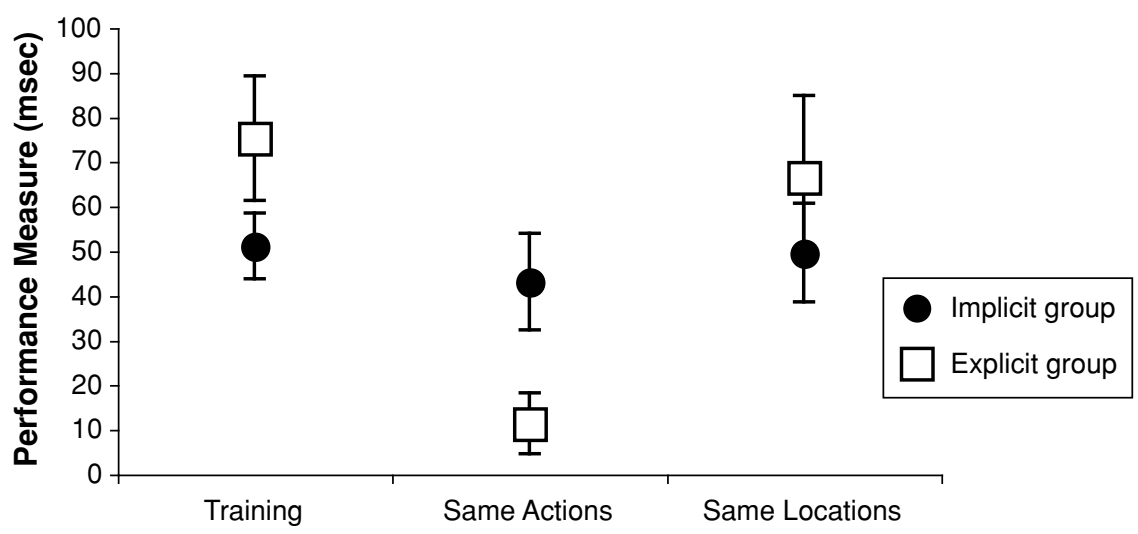

Figure 3. Performance on the three test conditions separated by participants with only implicit knowledge and participants with explicit knowledge of the locations sequence. The performance measure is the response time on the sequenced stimuli subtracted from response time on the random stimuli within the same block. Error bars depict standard errors of the means.

than performance on the training sequence $[F(1,13)=$ 23.70, $\left.M S_{\mathrm{e}}=2,410, p<.001\right]$ and the same-locations sequence $\left[F(1,13)=12.67, M S_{\mathrm{e}}=3,354, p<.01\right]$. There were no differences between the training sequence and the same-locations sequence $[F(1,13)=0.67, p=.43]$. Furthermore, the explicit-locations group did not appear to learn the sequence of actions at all. Performance on the same-actions sequence was not better than $0[t(13)=$ $1.66, p=.12]$, whereas performance on the training sequence and the same-locations sequence was better than $0[t(13)=5.40, p<.001$, and $t(13)=3.61, p<.01$, respectively]. Thus, the participants with explicit knowledge of the locations sequence showed learning of the sequence of response locations but not the sequence of actions. These results were most likely due to the fact that these participants gained explicit knowledge of the sequence of locations, not the actions.

\section{DISCUSSION}

Most investigations of the representational nature of motor sequencing have used buttonpressing as the behavioral response. In contrast, this experiment employed several types of motor responses with the various manipulanda positioned at different locations. We measured performance on three tests to assess the learning of the sequence of response locations, the sequence of response actions, and the combined sequence of locations and actions. The participants who only had implicit knowledge of the sequences showed a different pattern of results than did the participants who had explicit knowledge of the locations sequence. The explicit-locations group only showed learning of the sequence of locations, whereas the implicit group learned both the sequence of response actions and the sequence of response locations, and performance was just as good on individual sequences as on the combined sequence. This is the first demonstration that a sequence of actions can be learned implicitly.

\section{Participants With Implicit Knowledge}

An alternative explanation is that instead of learning a sequence of motor actions, the participants with implicit knowledge learned a sequence of perceptual manipulanda. Our data do not speak to this issue directly, but other research has demonstrated that perceptual learning is usually explicit (e.g., Knee, Thomason, Ashe, \& Willingham, in press; Willingham, 1999). Therefore, we think that the participants likely learned the sequence of actions themselves, rather than the sequence of perceptual manipulanda.

Thus, our findings suggest that there may be a distinct representation for motor skill learning of a sequence of actions and another one for learning a sequence of response locations, since a single representation of the sequence would have resulted in poor performance when the sequence of actions and locations were separated. If the participants had learned only a single sequence of the combination of actions and locations, then performance would have been impaired when one sequence was presented in isolation of the other. In contrast, performance was good when each of the individual sequences was presented, suggesting that the participants learned both sequences independently.

Simultaneous acquisition of both sequences - response locations and response actions - is indicative of parallel representations that are responsible for each component of the response. There are differences of opinion as to whether performance differences should be interpreted as being a product of different systems as opposed to different representations. One necessary (but not sufficient) criterion for proposing multiple systems is that there is functional and anatomical separability (Willingham \& Goedert, 2001). In other words, if one system is damaged, the other system should still be intact, which would result in double dissociations in behavior. There is no evidence from our results to suggest either a functional or an anatomical distinction. Thus, a conclusion of multiple systems would be premature, and we provisionally interpret 
these results as indicating separate sequence representations within a single implicit motor sequencing system.

An alternative explanation to multiple sequence representations is that the participants learned a single sequence of abstract relationships between elements rather than the surface sequences of locations and actions. For example, if the participants had learned the abstract sequence of ABAC, this would transfer to knowledge about a sequence of specific locations, such as far left, far right, far left, and middle left. The abstract sequence would also transfer to a sequence of specific actions, such as twist, pinch, twist, and switch. Therefore, knowledge of the abstract sequence could support good transfer even when only one surface sequence is present, and performance on one surface sequence would be just as good as performance on the other surface sequence, since they would both be based on the same knowledge.

Data from other experiments indicate, however, that implicit knowledge is typically not abstract but rather is tied to the particular surface features in which it was encoded. For example, Dominey, Lelekov, Ventre-Dominey, and Jeannerod (1998) observed abstract sequence learning only when participants were instructed to look for the abstract structure and when they successfully gained explicit knowledge of it. Participants with implicit knowledge only showed learning of the surface sequence and showed poor transfer on a new task that changed the surface sequence and maintained the abstract structure. Therefore, our results demonstrate that there are two types of representations in implicit motor sequencing: one for actions and one for locations.

The hypothesis of multiple representations for components of the response is consistent with findings on visuomotor transformations in the posterior parietal cortex. Spatial locations are encoded by different areas in the brain for reaching movements and for grasping movements (e.g., Colby, 1998). A number of researchers have suggested that motor skill learning is supported by the same neural structures that support motor control (e.g., Willingham, 1998). Thus, by analogy, motor sequences for reaching to spatial locations may be represented separately from motor sequences for forming specific grip postures.

\section{Multiple Systems for Implicit Learning}

Others have suggested that motor sequence learning is supported by multiple processes. In Mayr's (1996) SRT task, the stimuli appeared at different locations, but the stimulus shape cued the response. Both shape and location followed a sequence, and Mayr observed independent learning of both sequences. He concluded that one system learns a sequence of spatial orienting responses, and another system learns a sequence of motor responses. Keele, Ivry, Mayr, Hazeltine, and Heuer (2003) have also argued for two systems that learn sequences: one for sequences across multiple dimensions (e.g., vision and audition) and another for unidimensional sequences. These theories focus on the stimulus characteristics, whereas our results indicate that there may be multiple representations for sequenced response selection - specifically, one for learning where to respond and one for learning how to respond.

Our proposal raises the question of how the two representations interact. Previous research on parallel systems has often demonstrated additive effects. For example, performance on the combined sequence of spatial and nonspatial stimuli was as good as the summed performance on the individual sequences (Mayr, 1996). However, our results showed that performance on the combined sequence of response actions and response locations was the same as performance on each of the individual sequences.

One possible explanation for why performance was not better when both sequences were presented than when just one sequence was presented is that the participants acquired two types of knowledge: They learned both sequences in parallel, and they also learned the relationship between each individual manipulandum and its location. Participants associate, for example, the left side of the response board with the pinching response. When they anticipate pinching, they can also anticipate responding to the far left side of the response board. Because these two sources of information are perfectly correlated, one can substitute one for the other, and having both confers no advantage. Thus, if one knows the location of each manipulandum, knowing either the sequence of response locations or the sequence of actions is functionally equivalent to knowing both sequences. Therefore, performance would be just as good when individual sequences were presented as when both sequences were presented at the same time. According to this interpretation, the representations may interact indirectly with each other via additional knowledge about the layout of the response board.

\section{Participants With Explicit Knowledge}

The participants with explicit knowledge of the locations sequence showed learning when the sequence of response locations was held constant. However, the stimulus sequence and the response location sequence were confounded, so an alternative explanation is that the participants learned the sequence of perceptual stimuli. Our data are not decisive on this point, but other data from our lab indicate that when explicit learning occurs in the standard SRT paradigm, it is usually perceptual (Knee et al., in press).

The participants with explicit knowledge of the locations (or stimulus) sequence did not demonstrate learning of the sequence of actions. Given that the implicit group learned both location and action sequences, it is surprising that the explicit-locations group did not demonstrate learning the sequence of actions. This result conflicts with previous research that has demonstrated that explicit knowledge does not interfere with implicit acquisition of sequences (Bischoff-Grethe, Goedert, Willingham, \& Grafton, 2004; Mayr, 1996; Willingham \& GoedertEschmann, 1999; Willingham, Salidis, \& Gabrieli, 2002). Given these prior findings, we think it improbable that 
explicit knowledge of the stimulus sequence interfered with implicit acquisition of the action sequence. Rather, the explicit-locations group's poor performance on the sequence of actions may reflect a problem of expression. These participants knew the stimulus sequence and so might have sought to use it at transfer; the sequence of stimuli was no longer present, however, and that may have interfered with their performance.

In summary, we have demonstrated that a sequence of distinct actions can be learned implicitly. Many types of motor skills, such as dancing and martial arts, require learning a sequence of actions. Our findings suggest an independent representation for this type of motor skill learning. Previous theories argue that there are multiple systems for processing inputs; our results suggest multiple representations for learning the components of the response.

\section{REFERENCES}

Bischoff-Grethe, A., Goedert, K. M., Willingham, D. T., \& GrafTON, S. T. (2004). Neural substrates of response-based sequence learning using fMRI. Journal of Cognitive Neuroscience, 16, 127-138.

ColBY, C. L. (1998). Action-oriented spatial reference frames in cortex. Neuron, 20, 15-24.

Dominey, P., Lelekov, T., Ventre-Dominey, J., \& Jeannerod, M. (1998). Dissociable processes for learning the surface structure and abstract structure of sensorimotor sequences. Journal of Cognitive Neuroscience, 10, 734-751.

Keele, S. W., Ivry, R., Mayr, U., Hazeltine, E., \& Heuer, H. (2003). The cognitive and neural architecture of sequence representation. $P s y$ chological Review, 110, 316-339.

Knee, R., Thomason, S., Ashe, J., \& Willingham, D. T. (in press). The representation of explicit motor sequence knowledge. Memory \& Cognition.

MAYR, U. (1996). Spatial attention and implicit sequence learning: Evidence for independent learning of spatial and nonspatial sequences. Journal of Experimental Psychology: Learning, Memory, \& Cognition, 22, 350-364.
Nattkemper, D., \& Prinz, W. (1997). Stimulus and response anticipation in a serial reaction time task. Psychological Research, 60, 98112.

Nissen, M. J., \& Bullemer, P. (1987). Attentional requirements of learning: Evidence from performance measures. Cognitive Psychology, 19, 1-32.

Remillard, G. (2003). Pure perceptual-based sequence learning. Journal of Experimental Psychology: Learning, Memory, \& Cognition, 29, 581-597.

Squire, L. R., \& Zola-Morgan, S. (1991). The medial temporal lobe memory system. Science, 253, 1380-1386.

WillinghaM, D. B. (1998). A neuropsychological theory of motor skill learning. Psychological Review, 105, 558-584.

WillinghaM, D. B. (1999). Implicit motor sequence learning is not purely perceptual. Memory \& Cognition, 27, 561-572.

Willingham, D. B., \& Goedert, K. (2001). The role of taxonomies in the study of human memory. Cognitive, Affective, \& Behavioral Neuroscience, 1, 250-265.

Willingham, D. B., \& Goedert-Eschmann, K. M. (1999). The relation between implicit and explicit learning: Evidence for parallel development. Psychological Science, 10, 531-534.

Willingham, D. B., Nissen, M. J., \& Bullemer, P. (1989). On the development of procedural knowledge. Journal of Experimental Psychology: Learning, Memory, \& Cognition, 15, 1047-1060.

Willingham, D. B., Salidis, J., \& Gabrieli, J. D. E. (2002). Direct comparison of neural systems mediating conscious and unconscious skill learning. Journal of Neurophysiology, 88, 1451-1460.

Willingham, D. B., Wells, L. A., Farrell, J. M., \& Stemwedel, M. E. (2000). Implicit motor sequence learning is represented in response locations. Memory \& Cognition, 28, 366-375.

\section{NOTE}

1. In addition to the analyses reported here in which our recall cutoff was at 6 or more units, we also divided the participants into implicit- and explicit-locations groups, with a recall cutoff at 5 and at 7 . Using these different cutoffs did not qualitatively change the pattern of results.

(Manuscript received April 1, 2005; revision accepted for publication February 17, 2006.) 